\title{
Arm-wrestling injuries - A systematic review of the medical literature
}

\author{
Renan Felipe Correia', Alex Natalino Ribeiro', Roberto Pires de Araújo'
}

\begin{abstract}
Background: Arm wrestling is a popular sport due to its simplistic nature and great appeal in popular culture. Unfortunately, it can lead to serious fractures. An understanding of these injuries and its mechanisms is vital to the undertaking of safe practice methods for this sporting modality. Objectives: This study aimed to review articles that demonstrate case reports about arm-wrestling related fractures. Methods: PubMed's database was searched with the keywords "Arm Wrestling," Arm-wrestling," and "Armwrestling." Of the 34 articles found, 11 were fit to compose this review, being grouped into studies that dealt with adults and studies that dealt with adolescents. Results: Of the 63 adult subjects reported, 62 suffered fractures of the distal third of the humerus, occasionally accompanied by butterfly fractures and radial nerve palsy. Of the 22 adolescent subjects reported, 20 suffered fractures of the humerus medial epicondyle, never accompanied by butterfly fractures, and sometimes accompanied by ulnar nerve palsy. All injuries stemmed from unbalanced torsional forces suffered by the humerus from its own musculature. Conclusions: The injury profile presented by both populations is very homogeneous between the two different groups, which leads us to believe that correct technique can be a great ally in arm wrestling injury prevention. Athletes, as well as common practitioners, should be coached as to avoid these unfavorable positions and techniques Keywords: Arm Wrestling; Fractures; Injuries
\end{abstract}

\section{INTRODUCTION}

Arm wrestling is a very simple and popular sport due to its minimalist nature and great appeal in popular culture ${ }^{(1)}$. Unfortunately, it can be the catalyst of serious fractures which have been reported in the relevant medical literature ${ }^{(2)}$. Reports on injuries during arm wrestling are increasing, with fractures of the humerus shaft being the most common in nature ${ }^{(3)}$. During an arm wrestling match, two athletes face each other, either standing or sitting down, tie hands together and try to pin their opponents arm down to specified horizontal plane. These arm torsions, combined with other factors, are likely the key motives for fractures in arm wreslting ${ }^{(1)}$. An understanding of these injuries and its mechanisms is vital to the undertaking of safe practice methods for this sporting modality ${ }^{(4-6)}$.

\section{METHODOLOGY}

Given the increasing number of arm-wrestling related injuries present in the specific medical literature ${ }^{(7)}$, this study aimed to review articles that demonstrate case reports about this specific topic according to the following criteria:

1. Be a medical case report with human subjects (thus excluding cadaveric, computational, or machine models)
2.Be specific to injuries sustained during the practice of arm wrestling

3. Be written in English and published in peer-reviewed journals

Article search occurred in the PubMed database with the keywords "Arm Wrestling," Arm-wrestling," and "Armwrestling." Our primary search found 34 articles. Title and abstract inspection of these articles resulted in the exclusion of 21 titles. A full reading of the remaining 13 articles resulted in the further exclusion of three more articles, due to the use of cadaveric or computational models only. Thus, 11 articles were considered fit to compose this review.

\section{RESULTS}

\section{Subjects}

Of the 11 articles reviewed, seven presented case reports dealing strictly with adult subjects over the age of 18 years-old, totaling 63 subjects in total. Meanwhile, three articles presented case reports dealing strictly with adolescent subjects less than 18 years-old, totaling 22 subjects in total. Only one article did not discriminate subjects based on age.

Corresponding Author: Renan Felipe Correia. Adress: Av. Érico Veríssimo, 701 - Barão Geraldo, Campinas (SP), Brazil. 13083-851. E-mail: renanfcorreia@gmail.com Telephone Number: 5519993132881

${ }^{1}$ Department of Physical Education and Humanities. College of Physical Education at the Universidade de Campinas (UNICAMP), Campinas (SP), Brazil. Financial Support: None

Submission date 02 February 2018; Acceptance date 02 May 2018 ; Publication date 02 July 2018 
Due to the specific nature of bone and muscle development related to biological and maturational age, we will likewise present our data in such manner. Of all the 85 subjects reported in the 11 articles, only two were women, making gender an impractical review/analysis category.

\section{Models}

Nine research papers presented multi-case studies, while two of them presented single-case studies.

\section{Types of Injuries}

Seven studies present in this review focused on adult subjects, totaling 63 cases of arm-wrestling related injuries. Of those, 62 presented spiral fractures of the distal third of the humerus (SFDH), while only one of them presented a fracture of the radial neck (RNF), showing a very homogenous injury site profile. In 22 (35\%) of the injuries, the SFDH were accompanied by butterfly fractures (BF), 14 (22.2\%) of them were accompanied by radial nerve palsy (RNP). This information is summed up in Table 1.

The four studies that dealt with adolescent subjects under 18 years of age showed a very different injury data profile. In all, 22 injury cases were reported in them, 20 (91\%) of them present humerus medial epicondyle fractures (MEF), and only two presenting SFDH. Only one reported case was accompanied by a BF, and three were accompanied by ulnar nerve palsy (UNP). This information is summed up in table 2.

Table 1 - Case-Report Studies with Adult Subjects (>18 yrs old)

\begin{tabular}{|c|c|c|c|c|}
\hline Study & $\mathrm{n}$ & Major Fracture & $\begin{array}{l}\text { Minor } \\
\text { Fracture }\end{array}$ & $\begin{array}{l}\text { Nerve } \\
\text { Palsy }\end{array}$ \\
\hline Bumbasirevic et al ${ }^{(2)}$ & 6 & $6 \mathrm{SFDH}$ & $1 \mathrm{BF}$ & - \\
\hline Rutkowska et al|(8) & 10 & $10 \mathrm{SFDH}$ & $5 \mathrm{BF}$ & 2 RNP \\
\hline Kruczyński et al ${ }^{(1)}$ & 9 & 9 SFDH & $4 \mathrm{BF}$ & 3 RNP \\
\hline Ogawa \& Ui(9) & 30 & $30 \mathrm{SFDH}$ & $7 \mathrm{BF}$ & 7 RNP \\
\hline Low \& $\operatorname{Lim}^{(10)}$ & 2 & $2 \mathrm{SFDH}$ & - & 2 RNP \\
\hline Falis et $\mathrm{al}^{(3)}$ & 1 & 1 RNF & - & - \\
\hline Whittaker ${ }^{(11)}$ & 5 & $5 \mathrm{SFDH}$ & $5 \mathrm{BF}$ & - \\
\hline Total & 63 & $\begin{array}{c}62 \mathrm{SFDH} \\
(98.5 \%), 1 \mathrm{RNF}\end{array}$ & 22 BF (35\%) & $\begin{array}{l}14 \mathrm{RNP} \\
(22.2 \%)\end{array}$ \\
\hline
\end{tabular}

Note: SFDH $=$ Spiral Fracture of the Distal Third of the Humerus, RFN = Radial Neck Fracture, $\mathrm{BF}=$ Butterfly Fracture, RNP = Radial Nerve Palsy

Table 2 - Case-Report Studies with Adolescent Subjects ( $<18$ yrs old)

\begin{tabular}{|c|c|c|c|c|}
\hline Study & n & Major Fracture & Minor Fracture & Nerve Palsy \\
\hline Low \& Lim, $1991^{(10)}$ & 3 & $1 \mathrm{SDHF}, 2 \mathrm{MEF}$ & - & 2 UNP \\
\hline Chang et al, 2000(12) & 1 & $1 \mathrm{SDHF}$ & $1 \mathrm{BF}$ & - \\
\hline Nyska et al, $1992^{(13)}$ & 8 & $8 \mathrm{MEF}$ & - & - \\
\hline Ogawa \& Ui, 1996(14) & 10 & $10 \mathrm{MEF}$ & - & 1 UNP \\
\hline Total & 22 & $\begin{array}{c}20 \mathrm{MEF}(91 \%) \\
2 \mathrm{SDHF}\end{array}$ & $1 \mathrm{BF}(4.5 \%)$ & $\begin{array}{l}3 \text { UNP } \\
(13.6 \%)\end{array}$ \\
\hline
\end{tabular}

Note: MEF = Humerus Medial Epicondyle Fracture, SDHF = Spiral Fracture of the Distal Third of the Humerus, BF = Butterfly Fracture, UNP = Ulnar Nerve Palsy

\section{DISCUSSION}

Humerus fractures resulting from violent muscular force is a well-documented injury in various sports where upper limb strength performance is determinant to success ${ }^{(15,16)}$. In arm wrestling, it is no different, its athletes being especially at risk to this kind of injury. In adults, fractures are usually spiral in nature and located at the distal third of the humerus, being potentially accompanied by butterfly fractures and radial nerve palsy. In adolescents, the characteristics of the injuries are different, being primarily avulsion fractures of the medial humerus epicondyle. In this case, butterfly fractures are never present, and UNP may be a possibility. According to Whitaker ${ }^{(11)}$, in adults the forces acting on the humerus while the forearm muscles are contracted during a match are bending (from arm abductors), axial compression (from arm flexors and shoulder adductors), and torsional (internal rotators of the arm). They exert tension on the humerus when the internal rotator muscles of the shoulder go from their maximum concentric contraction to passive eccentric contraction, resulting in an intense rotational force leading to the fracture ${ }^{(9,18)}$, usually when trying to escape from a losing position while keeping the glenoumeral joint fixed. Also, Nyska et al ${ }^{(13)}$, in relation to the morphological characteristics of the humerus, state that these forces may lead to a fracture of specifically the distal third of the bone due to it being the portion of the bone with the smallest circumference, therefore having the lowest moment of inertia, and also having the thinnest diaphyseal-metaphyseal junction.

Ogawa e $\mathrm{Ui}^{(14)}$ state that the primary reason for the difference between usual injury sites in adults and adolescents is that the latter's humerus growth plates are not yet closed, configuring themselves as weak points where arm flexor musculature tension may be greater than the strength of the growth plate itself, thus acting in its fracture on violent muscle contractions ${ }^{(17,14,19,20)}$. Due to the presence of this weak spot, an avulsion fracture in the medial humeral epicondyle happens before the tensional forces can even reach the shaft of the humerus.

\section{FINAL CONSIDERATIONS}

Arm wrestling is a popular activity, which should not be perceived as safe when practiced by amateurs, as many reports have proven such ${ }^{(21)}$. However, the homogenous nature of the reported injuries, together with the fact that fractures are rare in competitive, professional and supervised competitions ${ }^{(10)}$, leads us to believe that correct technique can be a great ally in arm wrestling injury prevention.

All articles reviewed in this study stated that injuries always occur when unbalanced tensional forces directly affect the humerus. In practice, this means a position when both the arm and forearm are rotated, either internally or externally, in juxtaposition to each other, on a fixed glenoumeral joint. Therefore, athletes, as well as common practitioners, should be coached as to avoid these unfavorable positions and techniques ${ }^{(22,23)}$. 


\section{Ethical Procedures}

This research was not conducted with living subjects; therefore, it is exempt from approval by an ethics committee

\section{AUTHOR CONTRIBUTIONS}

RFC and RPA framed questions for the review. ANR and RFC identified relevant work. RFC and ANR assessed the quality of studies. RPA and RFC summarized the evidence. RFC, RPA, and ANR interpreted the findings. RFC and RPA drafted the final manuscript.

\section{CONFLICT OF INTEREST}

Nothing to declare.

\section{REFERENCES}

1. Kruczynski J, Jaszczur Nowicki J, Topolinski T, Srokowski G, Manko G, Chantsoulis $M$, et al. Radiological and biomechanical analysis of humeral fractures occurring during arm wrestling. Medical science monitor : international medical journal of experimental and clinical research. 2012;18(5):CR303-7.

2. Bumbasirevic MZ, Lesic AR, Andjelkovic SZ, Palibrk TD, Milutinovic SM. Fractures of the humerus during arm wrestling. Vojnosanitetski pregled. 2014;71(12):1144-6.

3. Fallis G, Ferguson K, Malcolm B. An unusual fracture in arm wrestling. The Physician and sportsmedicine. 1990;18(4):93-6.

4. Robertson GA, Wood AM. Fractures in sport: optimising their management and outcome. World journal of orthopedics. 2015;6(11):850-63.

5. Chaker A, Filipinsky J. [Sport-related spiral fractures of the humeral diaphysis are not simple injuries]. Rozhledy v chirurgii : mesicnik Ceskoslovenske chirurgicke spolecnosti. 2003;82(5):235-8.

6. Peltokallio P, Peltokallio V, Vaalasti T. Fractures of the humerus from muscular voilence in sport. The Journal of sports medicine and physical fitness. 1968;8(1):21-5.

7. Lee YS, Chou YH, Chiou HJ, Lai YC. Use of sonography in assessing elbow medial collateral ligament injury after arm wrestling. Journal of the Chinese Medical Association. 2014;77(3):163-5.

8. Frankowska-Rutkowska M, Górska J, Jedwabiński M, Mątewski D, Maciejewski M. Fracture of the humerus during arm wrestling: report on 9 cases and review of the literature. Medical and Biological Sciences. 2013;27(3):36-38.
9. Ogawa K, Ui M. Humeral shaft fracture sustained during arm wrestling: report on 30 cases and review of the literature. The Journal of trauma. 1997;42(2):243-6.

10. Low BY, Lim J. Fracture of humerus during armwrestling: report of 5 cases. Singapore medical journal. 1991;32(1):47-9.

11. Whitaker JH. Arm wrestling fractures--a humerus twist. The American journal of sports medicine. 1977;5(2):67-77.

12. I-Wen Chang S-CC, Jui-Sheng Sun. Humeral Shaft Fracture in Arm Wrestling. A Case Report. Journal of Emergency Medicine. 2000;2(2):5.

13. Nyska M, Peiser J, Lukiec F, Katz T, Liberman N. Avulsion fracture of the medial epicondyle caused by arm wrestling. The American journal of sports medicine. 1992;20(3):347-50.

14. Ogawa K, Ui M. Fracture-separation of the medial humeral epicondyle caused by arm wrestling. The Journal of trauma. 1996;41(3):494-7.

15. Hufner A, Dodt C. Spiral Fracture of the Humerus after a Tennis Serve. Deutsches Arzteblatt international. 2017;114(8):138.

16. Reed WJ, Mueller RW. Spiral fracture of the humerus in a ball thrower. The American journal of emergency medicine. 1998;16(3):306-8.

17. Koca K, Ege T, Kurklu M, Ekinci S, Bilgic S. Spiral-medial butterfly fractures (AO-12-B1) in distal diaphysis of humerus with rotational forces: preliminary results of open reduction and plate-screw fixation. European review for medical and pharmacological sciences. 2015;19(23):4494-7.

18. Ahamed NU, Sundaraj K, Ahmad B, Rahman M, Ali MA, Islam MA. Effects of anthropometric variables and electrode placement on the SEMG activity of the biceps brachii muscle during submaximal isometric contraction in arm wrestling. Biomedizinische Technik Biomedical engineering. 2013;58(5):475-88.

19. Oda T, Watanabe K. Bare medial epicondyle physeal fracture of the humerus: A case report. Journal of clinical orthopaedics and trauma. 2017;8(Suppl 1):S45-7.

20. Thiyam R, Lalchandani R. Tardy ulnar nerve palsy after fracture non-union medial epicondyle of humerus - An unusual case. Journal of clinical orthopaedics and trauma. 2015;6(2):137-9.

21. Marks W, Penkowski M, Stasiak M, Witkowski Z, Dabrowski T, Wieruszewski J, et al. Humeral fracture in arm wrestling: bone morphology as a permanent risk factor. Indications for safety measures in arm wrestling. The Journal of sports medicine and physical fitness. 2014;54(1):88-92.

22. Adams WM, Casa DJ, Drezner JA. Sport safety policy changes: saving lives and protecting athletes. Journal of athletic training. 2016;51(4):358-60.

23. Helm RH, Stuart P. Fracture of humerus during use of an arm wrestling machine. British medical journal. 1986;293(6562):1644. 